\title{
Inhibition of HIF-1 $\alpha$ restrains fracture healing via regulation of autophagy in a rat model
}

\author{
JUNJIE QIAO, JIANG HUANG, MENG ZHOU, GUANGLEI CAO and HUILIANG SHEN \\ Department of Orthopedics, Xuanwu Hospital, Capital Medical University, Beijing 100053, P.R. China
}

Received March 26, 2018; Accepted November 11, 2018

DOI: $10.3892 / \mathrm{etm} .2018 .7115$

\begin{abstract}
It has been demonstrated that bone fracture is associated with the activation of autophagy, and upregulation of autophagy could promote fracture healing. Previous study by our group demonstrated that activating the HIF-1 $\alpha$ pathway via administration of cobalt (II) chloride $\left(\mathrm{CoCl}_{2}\right)$ could promote fracture healing in vivo. However, the role of hypoxia-inducible factor-1 $\alpha$ (HIF-1 $\alpha)$ in autophagy remains unknown. In the current study, rats were divided into two groups following tibial fracture and treated with echinomycin or dimethyl sulfoxide (DMSO). Rats were sacrificed at 7, 14, 28 and 42 days after fracture. The evaluation of fracture healing was performed by micro-computed tomography. In addition, the effects of echinomycin on microtubule-associated protein 1 light chain 3 (LC3 II), runt-related transcription factor 2 (Runx2), alkaline phosphatase (ALP), Unc-51-like autophagy activating kinase 1 (ULK1) and P62 were detected at the mRNA and protein levels by reverse transcription-quantitative polymerase chain reaction, western blotting and immunohistochemistry. The results demonstrated that the expression of LC3 II was markedly decreased following systemic administration of echinomycin ( $0.05 \mathrm{mg} / \mathrm{kg}$ every other day for 42 days, intraperitoneally). Furthermore, the levels of Runx2, ALP and ULK1 were decreased, while those of P62 were increased, at the mRNA and protein levels in rats treated with echinomycin in vivo. In
\end{abstract}

Correspondence to: Professor Huiliang Shen, Department of Orthopedics, Xuanwu Hospital, Capital Medical University, 45 Changchun Street, Xicheng, Beijing 100053, P.R. China

E-mail: shen_hui_liang@sina.com

Abbreviations: HIF-1 $\alpha$, hypoxia-inducible factor-1 $\alpha$; DMSO, dimethyl sulfoxide; ROI, region of interest; BV/TV, bone volume/total volume; BMD, bone mineral density; ALP, alkaline phosphatase; Runx2, runt-related transcription factor 2; VEGF, vascular endothelial growth factor; LC3 II, microtubule-associated protein 1 light chain 3; ULK1, Unc-51-like autophagy activating kinase 1; micro-CT, micro-computed tomography; IHC, immunohistochemistry; qPCR, quantitative polymerase chain reaction; PBS, phosphate-buffered saline

Key words: hypoxia-inducible factor- $1 \alpha$, echinomycin, fracture, autophagy summary, the current study suggested that HIF-1 $\alpha$ may serve an important role in fracture healing via the downregulation of autophagy.

\section{Introduction}

Fracture repair is a multi-staged and complex process, the end goal of which is the recovery of the functional and biomechanical state (1). A variety of biological factors influencing the outcome of fracture healing have been identified in recent decades $(2,3)$. Bone fracture may immediately sever local vasculature and result in the formation of a hematoma, which leads to low oxygen tension and regional hypoxia (4). As a result, hypoxia-inducible factor- $1 \alpha(\mathrm{HIF}-1 \alpha)$ may be upregulated at the fracture site. Recently, numerous studies have focused on the function of HIF-1 $\alpha$ in fracture healing $(5,6)$.

Previous work by our group identified that HIF-1 $\alpha$ could be induced by hypoxia in the early stages of bone regeneration (7). Vascular endothelial growth factor (VEGF) induced by HIF-1 $\alpha$ promotes angiogenesis, which contributes to intramembranous ossification adjacent to the fracture site, endochondral ossification at the fracture site, and the ensuing remodeling phase of fracture repair (8). Autophagy, as a cell degradation process, targets dysfunctional cytosolic macromolecules, membranes and organelles, which serve an important role in energy and nutrient regulation (9). A previous study has demonstrated that activation of autophagy may protect organs and tissues against infarction injury, including neurodegeneration, cardiomyopathies and abnormal skeletal development (10). It has been demonstrated that fractures may impair cellular homeostasis and place bone cells under considerable stress, which may lead to the activation of autophagy and promote fracture healing in turn $(11,12)$.

An improved understanding of HIF-1 $\alpha$ and autophagy in fracture healing may aid the development of novel potential therapies for fracture healing. Therefore, the aim of the present study was to investigate whether autophagy is involved in fracture healing and the potential role of HIF-1 $\alpha$ in autophagy during fracture healing.

\section{Materials and methods}

Animal care and experiments. All experimental procedures involving rats were approved by the Ethics Committee on Animal Experimentation of Capital Medical University 
(Beijing, China; approval no. AEEI-2017-098). A total of

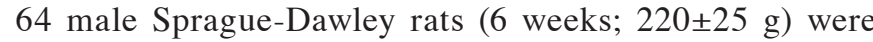
obtained from the Laboratory Animal Center of Capital Medical University. Upon arrival at the central animal facility, rats were allowed 1 week to acclimatize prior to random assignment into two groups: A control group treated with dimethyl sulfoxide (DMSO; $\mathrm{n}=32$ ); and an experimental group treated with $0.05 \mathrm{mg} / \mathrm{kg}$ echinomycin $(\mathrm{n}=32)$. Each group was treated intraperitoneally every other day for a total of 42 days. Subsequent to peritoneal injection of $1 \%$ sodium pentobarbital (40 mg/kg body weight; cat. no. P3761; Sigma-Aldrich; Merck KGaA, Darmstadt, Germany), all rats underwent fracture of the left tibia, which was performed with a blunt guillotine apparatus driven by a drop weight, as previously described (12). To achieve intramedullary fixation, a $0.8 \mathrm{~mm}$ Kirschner wire (K-wire) was inserted through the intercondylar notch until it was seated in the distal cortex. Radiographs were obtained immediately to confirm K-wire placement as well as the extent of fractures. Rats were sacrificed at 7, 14, 28 and 42 days after fracture ( $n=8 /$ group at each time point) by cervical dislocation following anesthesia by peritoneal injection of $1 \%$ sodium pentobarbital (40 $\mathrm{mg} / \mathrm{kg}$ body weight).

Micro-computed tomography (micro-CT) analysis. K-wires in the tibia were removed carefully in order to protect the fracture site, and then an Inveon micro-CT (Siemens AG, Munich, Germany) was used to scan the dissected tibia. The beam protocol was set as follows: $15-\mathrm{mm}$ isometric voxel size, $800 \mathrm{~mA}$ and $80 \mathrm{kV}$. The proximal and distal bone tissue that was $2.5 \mathrm{~mm}$ far away from the fracture line was selected as the region of interest (ROI). The callus perimeter was determined by a semi-automated contouring method. Contours were drawn to reveal the periosteal surface of the ROI in the tibia. The tibiae were reconstructed using Mimics software version 20.0 (Materialise NV, Leuven, Belgium), and bone histomorphometric analysis, including of bone mineral density (BMD) and bone volume fraction [bone volume/total volume (BV/TV)], was performed using the built-in software in the micro-CT system (data not shown) (13).

RNA extraction and reverse transcription-quantitative polymerase chain reaction ( $q P C R)$. Total RNA was isolated from the callus using TRIzol (Invitrogen; Thermo Fisher Scientific, Inc., Waltham, MA, USA) according to the manufacturer's protocol. Then, cDNA was reverse transcribed using a ReverTra Ace kit (Toyobo Life Science, Osaka, Japan). qPCR was performed to measure mRNA levels relative to GAPDH expression. The sequences of the primers used were as follows: For runt-related transcription factor 2 (Runx2), forward, 5'-CCCACGAATGCA CTATCCAG-3' and reverse, 5'-GGCTTCCATCAGCGTCAA CA-3'; for alkaline phosphatase (ALP), forward, 5'-GGACGG TGAACGGGAGAAC-3' and reverse, 5'-CCCTCAGAACAG GGTGCGTAG-3'; for microtubule-associated protein 1 light chain 3 (LC3 II) forward, 5'-AAACGCATTTGCCATCACA-3 and reverse, 5'-CCCTCAGAACAGGGTGCGTAG-3'; for P62, forward, 5'-GGGGACTTGGTTGCCTTTT-3' and reverse, 5'-CAGCCATCGCAGATCACATT-3'; and for Unc-51-like autophagy activating kinase 1 (ULK1), forward, 5'-TCGAGT TCTCCCGCAAGG-3' and reverse, 5'-CGTCTGAGACTT GGCGAGGT-3'. The PCR conditions were set as follows: 3 min at $95^{\circ} \mathrm{C}$, followed by 40 cycles at $95^{\circ} \mathrm{C}$ for $15 \mathrm{sec}, 55-60^{\circ} \mathrm{C}$ for $30 \mathrm{sec}$ and $72^{\circ} \mathrm{C}$ for $1 \mathrm{~min}$, then $72^{\circ} \mathrm{C}$ for $10 \mathrm{~min}$ as a final extension. The expression values were normalized against GAPDH using the $2^{-\Delta \Delta C q}$ method (14). In order to minimize confounding variance, two independent samples were analyzed in technical triplicates. Technical replicates were averaged prior to all software analysis.

Immunohistochemistry (IHC). Sections were prepared and processed using standard techniques, as described previously (15). Briefly, tissues from the tibia were decalcified and paraffin embedded in preparation for tartrate-resistant acid phosphatase staining. Following antigen retrieval and blocking of endogenous peroxide activity, the slides were incubated with primary antibodies against ALP (cat. no. ab84401; Abcam, Cambridge, MA, USA; dilution 1:100), Runx2 (cat. no. H00000860-M04; Abnova, Taipei, Taiwan; dilution 1:100) and LC3 II (cat. no. ab48394; Abcam; dilution 1:100) in a humidified chamber at $4^{\circ} \mathrm{C}$ overnight. Subsequently, sections were incubated with goat anti-rabbit immunoglobulin $\mathrm{G}$ secondary antibody (cat. no. ab6720; Abcam; dilution 1:100) for $10 \mathrm{~min}$ at $37^{\circ} \mathrm{C}$. Following washing with phosphate-buffered saline (PBS), the sections were incubated with streptavidin-peroxidase conjugate (Rockland Immunochemicals, Inc., Limerick, PA, USA) for $10 \mathrm{~min}$ at room temperature. Final staining was performed using diaminobenzidine tetrahydrochloride solution (Ventana Medical Systems, Inc., Tucson, AZ, USA) and sections were counterstained with hematoxylin for $10 \mathrm{~min}$ at room temperature, and then mounted. Scores were assessed by two independent observers and evaluated according to the percentage of positively stained cells and intensity of staining. Immunostaining intensity was rated as follows: 0 , negative; 1 , weak; 2 , moderate; 3 , strong. The percentage of positively stained cells was rated as follows: Grade $0,<25 \%$; grade 1 , $<50 \%$; grade $2,<75 \%$; and grade $3,>75 \%$.

Western blot analysis. Dissected calluses from fractured bone for each time point (7, 14, 28 and 48 days) were washed with PBS. Tissue lysates were prepared with lysis buffer containing protease inhibitors (Sigma-Aldrich; Merck KGaA). Protein quantification was performed using a bicinchoninic acid protein assay kit (Thermo Fisher Scientific, Inc.). Lysates (30 mg) were separated on 10\% SDS-polyacrylamide gels and electrotransferred onto polyvinylidene difluoride membranes (EMD Millipore, Bedford, MA, USA). Membranes were blocked with $5 \%$ non-fat dry milk and incubated overnight at $4^{\circ} \mathrm{C}$ with primary antibodies against HIF-1 $\alpha$ (cat. no. ab463; Abcam; dilution 1:1,000), ULK1 (cat. no. ab128859; Abcam; dilution 1:1,000), p62 (cat. no. ab96134; Abcam; dilution $1: 1,000)$ and those aforementioned against ALP (dilution 1:2,000), Runx2 (dilution 1:500) and LC3 II (dilution 1:2,000). Membranes were then incubated with horseradish peroxidase-conjugated secondary antibodies (cat. no. ab97200, Abcam; dilution 1:5,000) for $2 \mathrm{~h}$ at room temperature. Bands were developed using chemiluminescence reagents (Thermo Fisher Scientific, Inc.) and densitometric results were analyzed with Image Quant LAS4000 (GE Healthcare Life Sciences, Little Chalfont, UK). GAPDH (cat. no. G5262; Sigma-Aldrich; Merck KGaA; dilution 1:5,000) served as a loading control. 
A
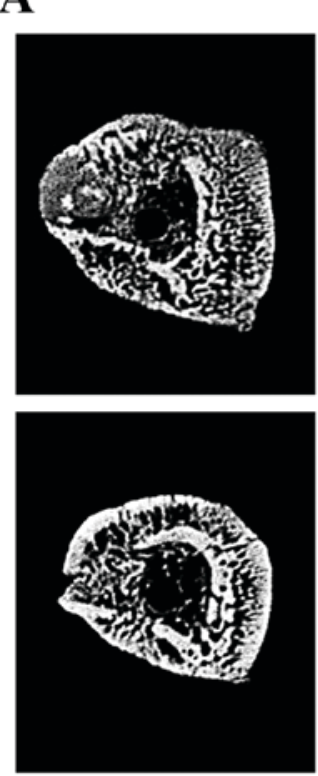

B
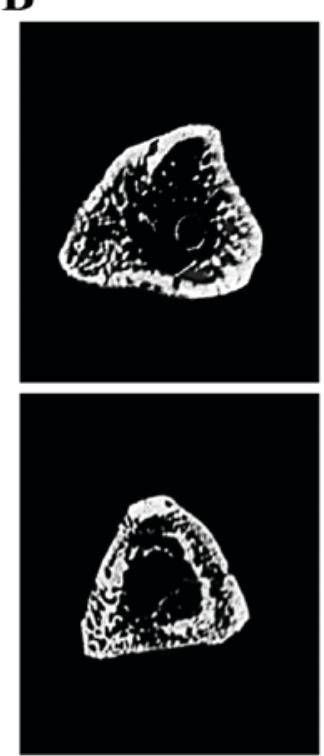

28 days
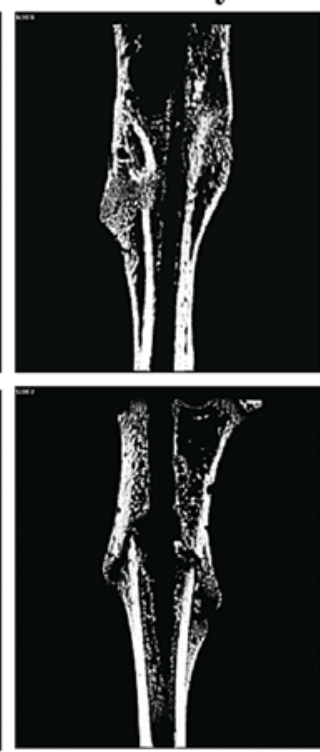

42 days
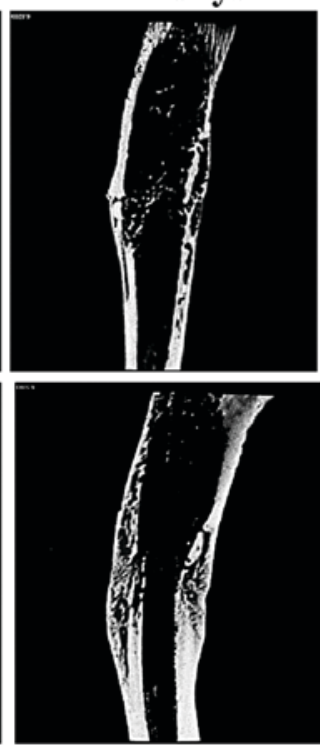

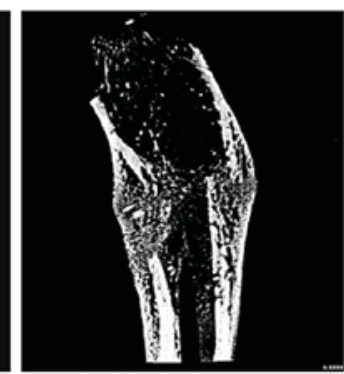

DMSO

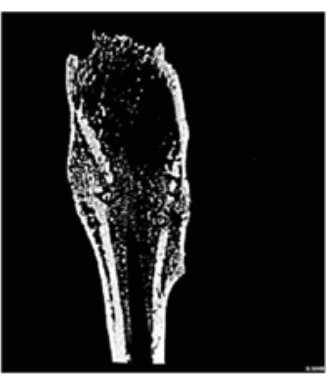

Echinomycin

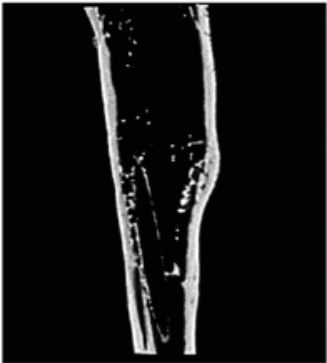

\section{DMSO}

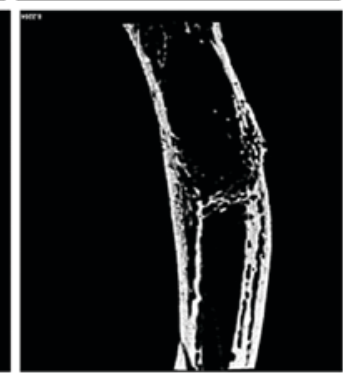

Figure 1. Micro-computed tomography images of the fracture callus at days 28 and 42 after fracture. Representative images of each group at (A) day 28 and (B) day 42 are presented. DMSO, dimethyl sulfoxide.

Statistical analysis. Each experiment was repeated at least three times. All statistical comparisons were performed with SPSS software, version 19.0 (IBM Corp., Armonk, IL, USA). Data are presented as the mean \pm standard deviation. The differences between the groups were analyzed by Student's t-test. $\mathrm{P}<0.05$ was considered to indicate a statistically significant difference.

\section{Results}

Echinomycin inhibits callus formation and remodelling. First, the differences in callus formation and remodeling between echinomycin and DMSO administration groups were compared. Micro-CT was used to remodel the fracture calluses and to quantify the degree of mineralization. The results demonstrated that treatment with echinomycin could markedly inhibit new bone formation and the subsequent remodeling process of fractured bone in rats. As a result, fractured tibiae exhibited delayed repair in echinomycin-treated rats compared with the control group. Notably, the control group displayed near-complete healing of the tibia at day 42 (Fig. 1). These results suggested that loss of function of HIF-1 $\alpha$ by echinomycin could inhibit fracture healing.

Echinomycin modulates HIF-1 $\alpha$ target genes and autophagy markers at the $m R N A$ level. Subsequently, the potential mechanism of inhibition of HIF-1 $\alpha$ in fracture healing was investigated. The mRNA levels of HIF-1 $\alpha$, its target-genes (osteoblast markers) and autophagy markers were assessed following echinomycin administration. It was identified that compared with in the DMSO group, the levels of ALP (Fig. 2A) and Runx2 (Fig. 2B) were significantly decreased following echinomycin administration. Furthermore, the levels 

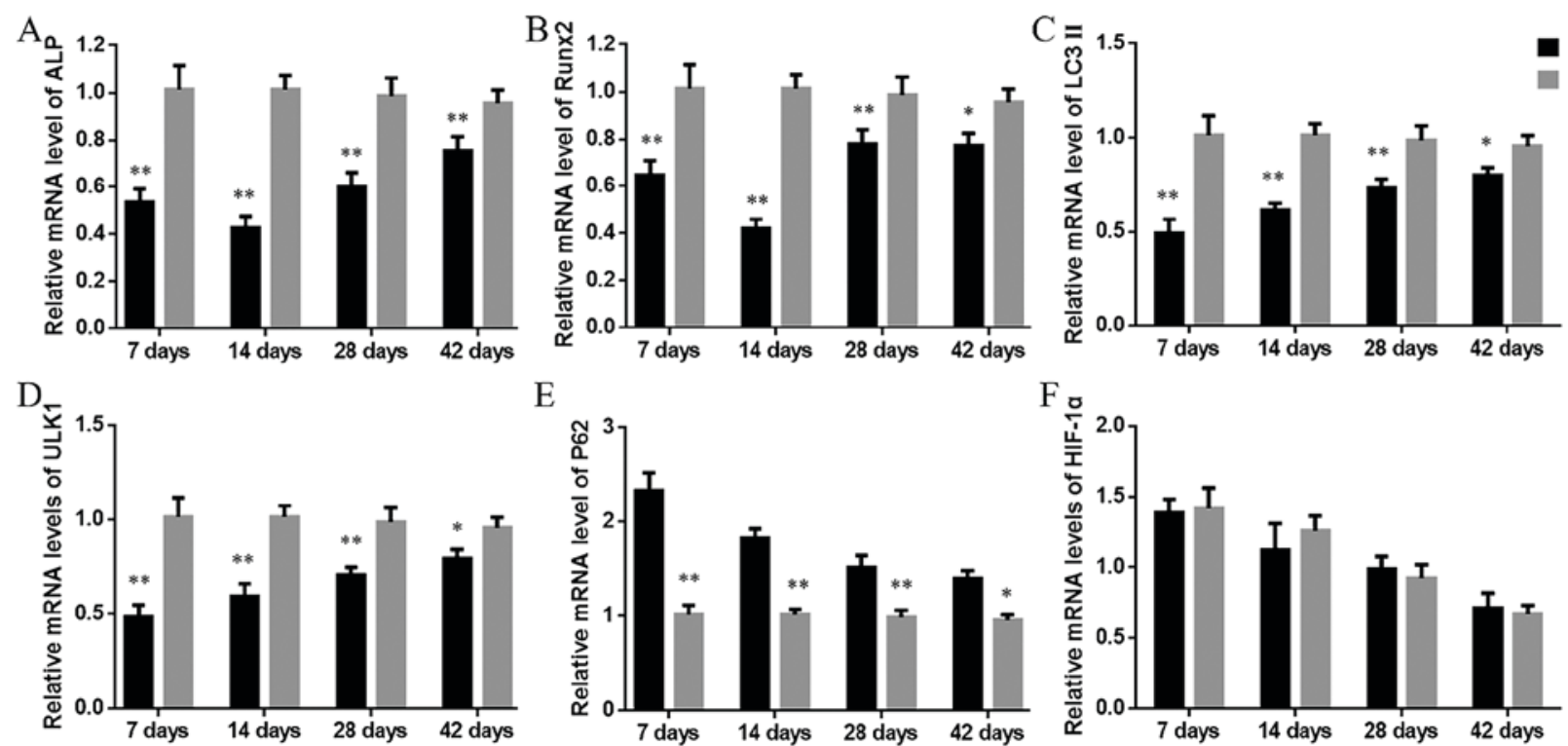

Figure 2. mRNA levels of (A) ALP, (B) Runx2, (C) LC3 II, (D) ULK1, (E) P62 and (F) HIF-1 $\alpha$ at the indicated time points. "P<0.05, **P $<0.01$ vs. DMSO-treated group. ALP, alkaline phosphatase; Runx2, runt-related transcription factor 2; LC3 II, microtubule-associated protein 1 light chain 3; ULK1, Unc-51 like autophagy activating kinase 1; HIF-1 $\alpha$, hypoxia-inducible factor-1 $\alpha$; DMSO, dimethyl sulfoxide.

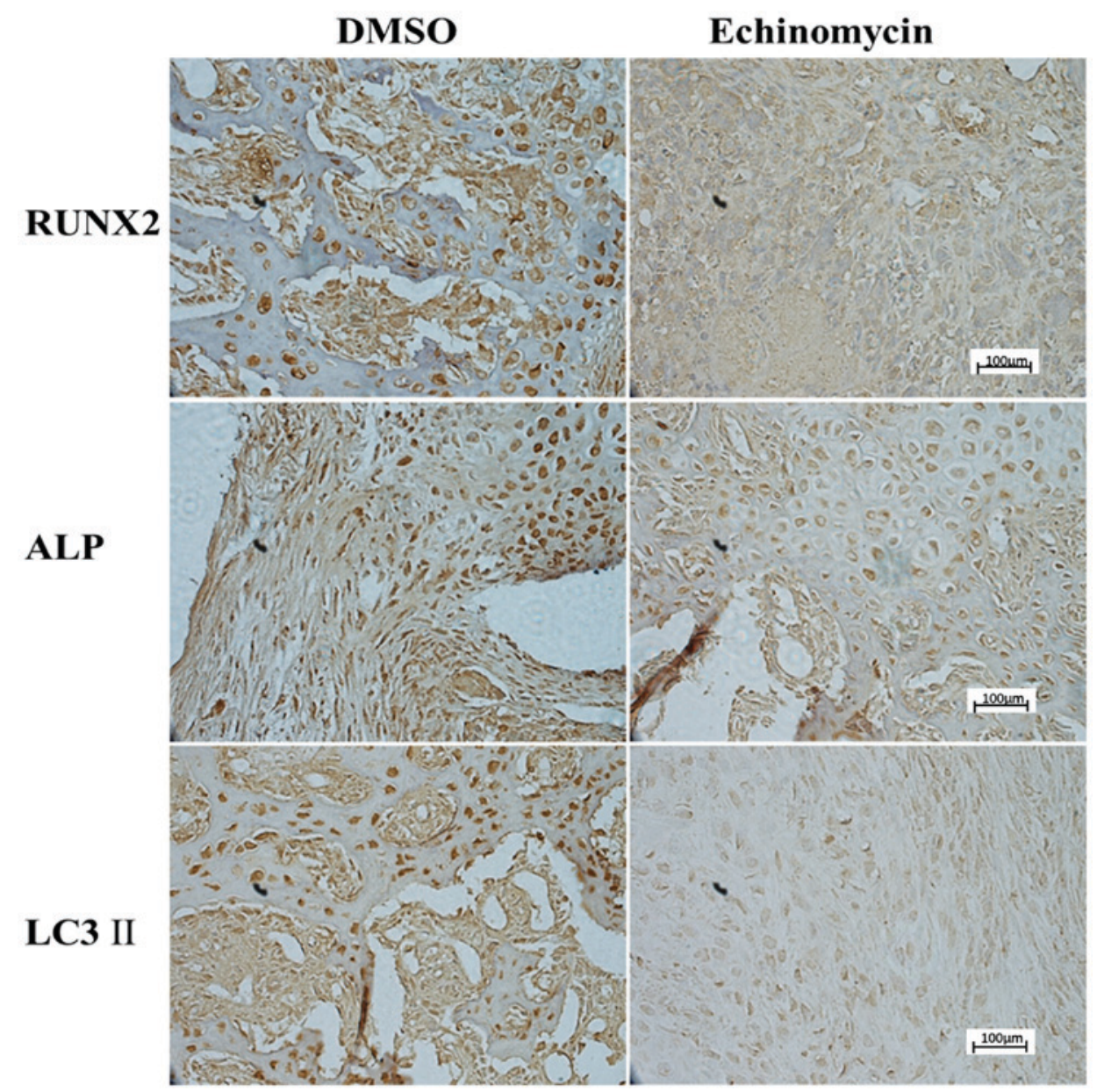

Figure 3. Representative images of immunohistochemical staining of Runx2, ALP and LC3 II in bone tissues at day 42 after fracture (magnification, $\mathrm{x} 400$ ). ALP, alkaline phosphatase; Runx2, runt-related transcription factor 2; LC3 II, microtubule-associated protein 1 light chain 3; DMSO, dimethyl sulfoxide.

of autophagy markers, LC3 II (Fig. 2C) and ULK1 (Fig. 2D) were also reduced in the echinomycin treatment group. The
mRNA levels of P62 were increased in the echinomycin treatment group (Fig. 2E), while HIF-1 $\alpha$ exhibited no significant 


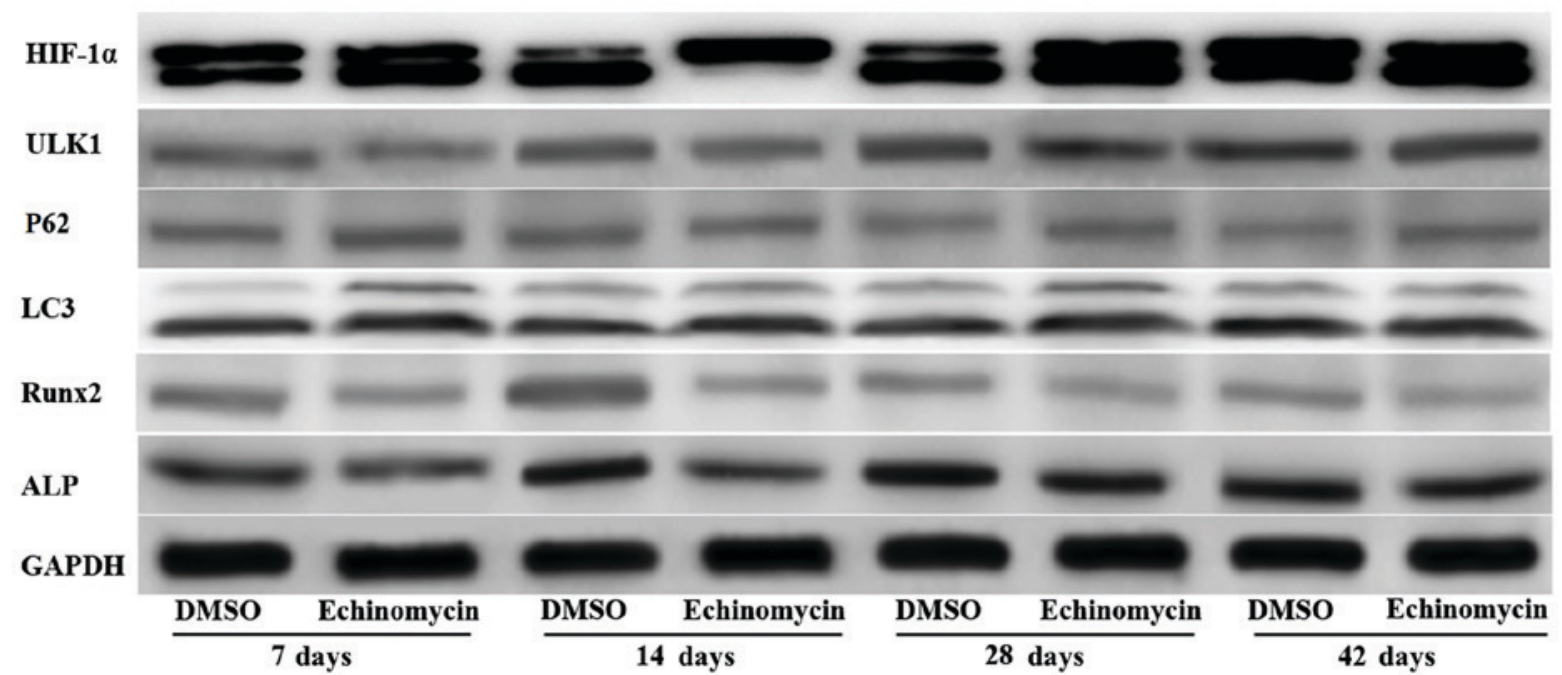

Figure 4. Western blot analysis of HIF-1 $\alpha$, ULK1, P62, LC3 II, Runx2 and ALP at the indicated time points after fracture. HIF-1 $\alpha$, hypoxia-inducible factor-1 $\alpha$; ULK1, Unc-51-like autophagy activating kinase 1; LC3 II, microtubule-associated protein 1 light chain 3; Runx2, runt-related transcription factor 2; ALP, alkaline phosphatase; DMSO, dimethyl sulfoxide.

difference in its expression at any time point between the two groups (Fig. 2F). These results indicated that loss of function of HIF- $1 \alpha$ by echinomycin may inhibit osteoblast formation and downregulate autophagy in fracture healing.

Echinomycin decreases the expression of HIF-1 $\alpha$ target genes and autophagy markers at the protein level. Protein levels of HIF-1 $\alpha$ target genes and molecular markers of autophagy were further evaluated by IHC and western blot analysis. As indicated in Fig. 3, rats with echinomycin administration exhibited a significant downregulation of Runx 2, ALP and LC3 II expression at day 42 after fracture. In addition, western blot analysis demonstrated that echinomycin treatment led to the significantly decreased expression of Runx2, ALP, ULK1 and slightly decreased the expression of LC3 II. The expression of P62 was also increased (Figs. 4 and 5). These results were consistent with those at the mRNA level, which further suggested a potential role of HIF-1 $\alpha$ in fracture healing.

\section{Discussion}

Fracture repair is a highly complex but well-orchestrated regenerative process involving specific gene expression and cellular recruitment (16). Fractures lead to inadequate blood supply and decreased $\mathrm{O}_{2}$ availability, which results in the upregulation of HIF-1 $\alpha$ (17). HIF-1 $\alpha$, an oxygen-sensitive transcription factor, mediates cellular responses to hypoxia during pathological processes in many diseases. Previous studies have demonstrated that an increase in HIF-1 $\alpha$ and its downstream cytokines is apparent in the process of fracture healing $(7,17)$.

When HIF-1 $\alpha$ interacts with the hypoxia response element (HRE) DNA sequence of a target gene, numerous hypoxia-sensitive genes are upregulated, including VEGF, heme oxygenase 1 and inducible nitric oxide synthase (18). Of note, it has been reported that HIF- $1 \alpha$ could enhance the expression of Runx2, which participates in the process of osteogenesis in vivo (7). Runx2 is a key regulator of bone formation via the regulation of osteoclast and osteoblast differentiation. By regulating the cell cycle of osteoblasts and the expression of specific extracellular matrix protein genes in osteoblasts, Runx 2 can promote bone formation and inhibit bone resorption (19). As a consequence, HIF-1 $\alpha$ is considered to aid in accelerating fracture healing (7). Echinomycin is an established inhibitor of HIF-1 $\alpha$, which acts by inhibiting its DNA-binding activity (20). In the current study, it was hypothesized that echinomycin may have an inhibitory role on fracture healing by downregulating the target genes of HIF-1 $\alpha$.

In this study, the expression of HIF-1 $\alpha$ was detected at the mRNA and protein levels in fracture calluses. It was identified that the levels of HIF-1 $\alpha$ did not differ significantly between the echinomycin and control groups throughout the fracture healing process, which was consistent with a previous study (21). However, Runx2 and ALP were significantly downregulated in the echinomycin group. A possible interpretation is that an increase of HIF- $1 \alpha$ was induced by the condition of hypoxia caused by fracture, which was the same in both groups, and echinomycin inhibited HIF-1 $\alpha$ by affecting the DNA-binding activity of HIF-1 $\alpha$ with its target genes, and not via affecting the expression and transcription of HIF-1 $\alpha$. Furthermore, micro-CT of the tibia demonstrated that the density and the amount of callus tissue between the echinomycin and control groups were different. The volume of callus in the echinomycin group was significantly increased compared with in the control group during the callus formation stage, and BMD was notably increased in the control group (data not shown). In summary, these results support the proposal that administration with echinomycin may delay the fracture healing by causing downregulating of the HIF-1 $\alpha$ target genes.

In recent years, the study of autophagy in bone research has been an emerging field. Autophagy is a catabolic process characterized by removal of defective organelles and mobilization of nutrients in order to maintain energy balance (22). Increasing evidence has demonstrated that energy status and nutrient levels are key regulatory factors of 

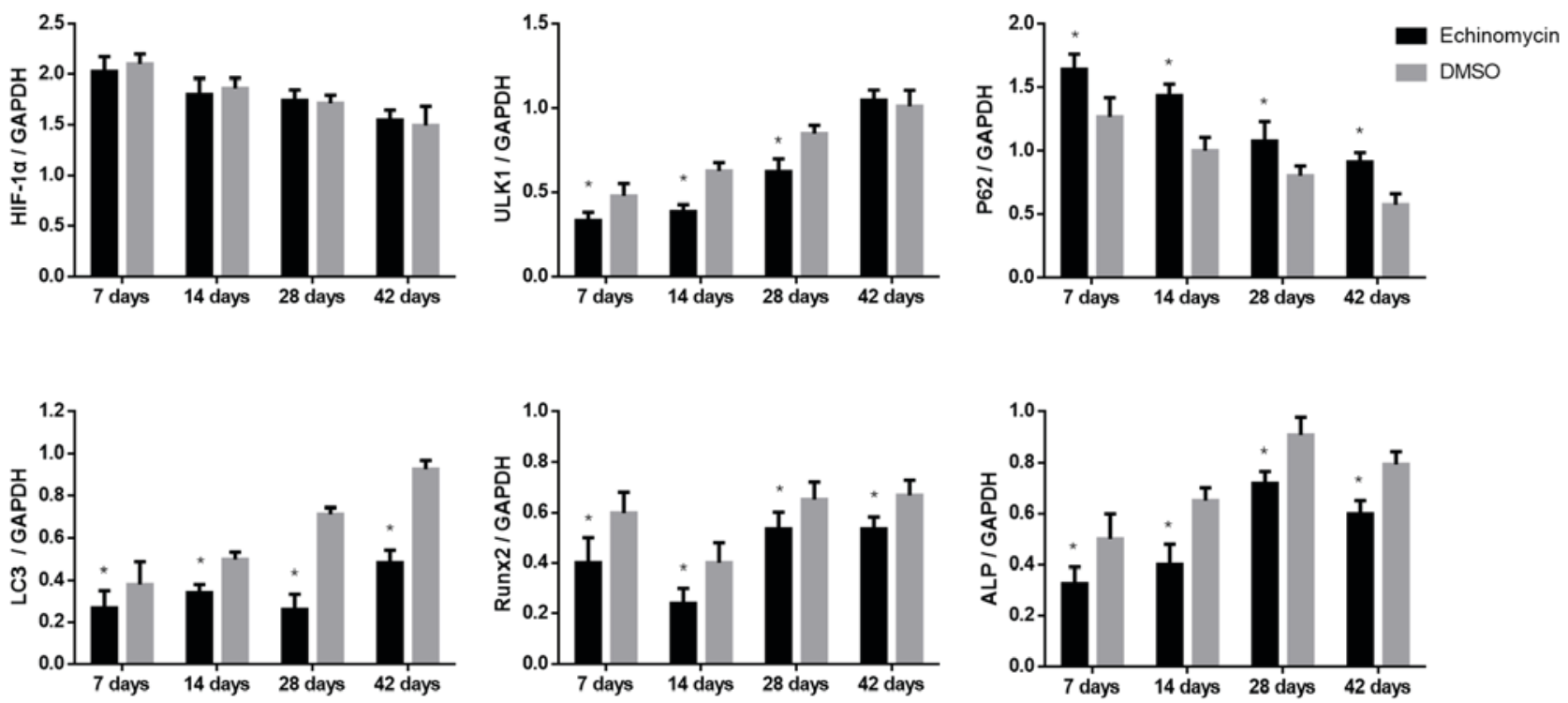

Figure 5. Quantitative analysis of HIF-1 $\alpha$, ULK1, P62, LC3 II, Runx2 and ALP protein expression at the indicated time points after fracture. * $<0.01$ vs. DMSO-treated group. HIF-1 $\alpha$, hypoxia-inducible factor-1 $\alpha$; ULK1, Unc-51-like autophagy activating kinase 1; LC3 II, microtubule-associated protein 1 light chain 3; Runx2, runt-related transcription factor 2; ALP, alkaline phosphatase; DMSO, dimethyl sulfoxide.

autophagy (23). Once the physiological balance is disrupted, autophagy may be triggered in cells to enable a response to the changing conditions $(23,24)$. Previous studies have demonstrated that autophagy is an HIF- $1 \alpha$-dependent adaptive metabolic response to hypoxia (25-27). However, it had thus far remained unclear how autophagy is altered if HIF-1 $\alpha$ is inhibited.

The present study provided experimental data to suggest that HIF-1 $\alpha$ may serve a critical role in the regulation of autophagy during the process of fracture healing. LC3 serves a critical role in the formation and elongation of the autophagosome and is among the most studied proteins in monitoring autophagic activity (28). In the current study, the levels of LC3 II were slightly downregulated in the echinomycin-treated group compared with in the control group. As previously described, ULK1 is enzymatically activated in response to nutrient deprivation and may phosphorylate autophagy-related protein 13 to initiate the process of autophagy (29). A disruption of blood supply by fracture may generate hypoxic conditions around the damaged area and promote anaerobic metabolism, which may give rise to insufficient nutrients and energy (2). Therefore, it is inferred that autophagy following fracture may be an energy-dependent process and regulated by the HIF-1 $\alpha$ pathway (23). P62, a multifunctional ubiquitin-binding protein, has been proposed to be involved in the degradation of target proteins and cytoplasmic bodies by lysosomal enzymes (30). When also considering the expression data on P62, which were the opposite to that of LC3 II and ULK1, the current results strongly indicated that autophagy was inhibited during the process of fracture healing in the echinomycin-treated group. The results were consistent with the view that autophagy is an important process utilized by HIF-1 $\alpha$ to maintain cell homeostasis (22). Combined with other recent studies $(26,31)$, these results support the conclusion that HIF-1 $\alpha$ and autophagy are linked. Although the association between HIF-1 $\alpha$ and autophagy requires further investigation, this suggests that maintenance of HIF-1 $\alpha$ and autophagy may serve a functional role in bone regeneration in fracture healing.

In conclusion, the present results demonstrate that HIF-1 $\alpha$, which serves an important role in fracture healing, may serve as a target to improve bone healing. Furthermore, the autophagy induced by fracture may be HIF-1 $\alpha$-dependent and HIF-1 $\alpha$ may also affect the healing process by regulating autophagy. These findings highlight the role of HIF-1 $\alpha$ and provide a potential strategy for the regeneration of bone tissue. However, determining the factor(s) utilized by HIF-1 $\alpha$ in the process of fracture healing remains a topic of further study. Future studies should investigate the regulatory mechanisms associated with HIF-1 $\alpha$ and autophagy in more depth, in order to facilitate the development of treatments aimed at improving fracture healing.

\section{Acknowledgements}

Not applicable.

\section{Funding}

The present study was supported by the National Natural Science Foundation of China (grant no. 81541135) and Capital's Funds for Health Improvement and Research (grant no. 2018-2-2012).

\section{Availability of data and materials}

The datasets used and/or analyzed during the current study are available from the corresponding author on reasonable request.

\section{Authors' contributions}

JQ performed the majority of the experiments and prepared the manuscript. JH performed the polymerase chain reaction 
and western blot analyses. MZ analyzed the data. GC and HS designed the experiments. All authors read and approved the final manuscript.

\section{Ethics approval and consent to participate}

The present study was approved by the Ethics Committee on Animal Experimentation of Capital Medical University, Beijing, China.

\section{Patient consent for publication}

Not applicable.

\section{Competing interests}

The authors declare that they have no competing interests.

\section{References}

1. Einhorn TA and Gerstenfeld LC: Fracture healing: Mechanisms and interventions. Nat Rev Rheumatol 11: 45-54, 2015.

2. Giannoudis PV, Jones E and Einhorn TA: Fracture healing and bone repair. Injury 42: 549-550, 2011.

3. Oryan A, Monazzah S and Bigham-Sadegh A: Bone injury and fracture healing biology. Biomed Environ Sci 28: 57-71, 2015.

4. Sang X, Wang Z, Qin T and Li Y: Elevated concentrations of hypoxia-inducible factor- $1 \alpha$ in patients with fracture and concomitant traumatic brain injury. Ann Clin Biochem 54: 584-592, 2017.

5. Liu P, Liu J, Xia K, Chen L and Wu X: Effect of leptin combined with $\mathrm{CoCl} 2$ on healing in Sprague Dawley Rat fracture model Sci Rep 6: 30754, 2016.

6. Sun G and Peng H: HIF-1 $\alpha$-induced microRNA-210 reduces hypoxia-induced osteoblast MG-63 cell apoptosis. Biosci Biotechnol Biochem 79: 1232-1239, 2015.

7. Huang J, Liu L, Feng M, An S, Zhou M, Li Z, Qi J and Shen H: Effect of $\mathrm{CoCl}(2)$ on fracture repair in a rat model of bone fracture. Mol Med Rep 12: 5951-5956, 2015.

8. Schorn L, Sproll C, Ommerborn M, Naujoks C, Kubler NR and Depprich R: Vertical bone regeneration using rhBMP-2 and VEGF. Head Face Med 13: 11, 2017.

9. Pierrefite-Carle V, Santucci-Darmanin S, Breuil V, Camuzard O and Carle GF: Autophagy in bone: Self-eating to stay in balance. Ageing Res Rev 24: 206-217, 2015.

10. Singh SR, Zech ATL, Geertz B, Reischmann-Dusener S, Osinska H, Prondzynski M, Kramer E, Meng Q, Redwood C, van der Velden $\mathrm{J}$, et al: Activation of autophagy ameliorates cardiomyopathy in mybpc3-targeted knockin mice. Circ Heart Fail 10: e004140, 2017.

11. Yang GE, Duan X, Lin D, Li T, Luo D, Wang L and Lian K: Rapamycin-induced autophagy activity promotes bone fracture healing in rats. Exp Ther Med 10: 1327-1333, 2015.

12. Zhou Q, Luo D, Li T, Liu Z, Zou W, Wang L, Lin D and Lian K: Bone fracture in a rat femoral fracture model is associated with the activation of autophagy. Exp Ther Med 10: 1675-1680, 2015.

13. Bouxsein ML, Boyd SK, Christiansen BA, Guldberg RE, Jepsen KJ and Müller R: Guidelines for assessment of bone microstructure in rodents using micro-computed tomography. J Bone Miner Res 25: 1468-1486, 2010.
14. Livak KJ and Schmittgen TD: Analysis of relative gene expression data using real-time quantitative PCR and the 2(-Delta Delta C(T)) method. Methods 25: 402-408, 2001.

15. Gerstenfeld LC, Wronski TJ, Hollinger JO and Einhorn TA: Application of histomorphometric methods to the study of bone repair. J Bone Miner Res 20: 1715-1722, 2005.

16. Loi F, Córdova LA, Pajarinen J, Lin TH, Yao Z and Goodman SB: Inflammation, fracture and bone repair. Bone 86: 119-130, 2016.

17. Li W, Wang K, Liu Z and Ding W: HIF-1 $\alpha$ change in serum and callus during fracture healing in ovariectomized mice. Int J Clin Exp Pathol 8: 117-126, 2015.

18. Komatsu DE and Hadjiargyrou M: Activation of the transcription factor HIF-1 and its target genes, VEGF, HO-1, iNOS, during fracture repair. Bone 34: 680-688, 2004.

19. Perez-Campo FM, Santurtun A, Garcia-Ibarbia C, Pascual MA, Valero C, Garces C, Sanudo C, Zarrabeitia MT and Riancho JA: Osterix and RUNX2 are transcriptional regulators of sclerostin in human bone. Calcif Tissue Int 99: 302-309, 2016.

20. Kong D, Park EJ, Stephen AG, Calvani M, Cardellina JH, Monks A, Fisher RJ, Shoemaker RH and Melillo G: Echinomycin, a small-molecule inhibitor of hypoxia-inducible factor-1 DNA-binding activity. Cancer Res 65: 9047-9055, 2005.

21. Wang Z, Zhang Z, Wu Y, Chen L, Luo Q, Zhang J, Chen J, Luo Z, Huang $X$ and Cheng Y: Effects of echinomycin on endothelin-2 expression and ovulation in immature rats primed with gonadotropins. Exp Mol Med 44: 615-621, 2012.

22. Zhang H, Bosch-Marce M, Shimoda LA, Tan YS, Baek JH, Wesley JB, Gonzalez FJ and Semenza GL: Mitochondrial autophagy is an HIF-1-dependent adaptive metabolic response to hypoxia. J Biol Chem 283: 10892-10903, 2008.

23. Parzych KR and Klionsky DJ: An overview of autophagy: Morphology, mechanism, and regulation. Antioxid Redox Signal 20: 460-473, 2014.

24. Kroemer G: Autophagy: A druggable process that is deregulated in aging and human disease. J Clin Invest 125: 1-4, 2015.

25. Tsuzuki T, Okada H, Cho H, Tsuji S, Nishigaki A, Yasuda K and Kanzaki H: Hypoxic stress simultaneously stimulates vascular endothelial growth factor via hypoxia-inducible factor-1 and inhibits stromal cell-derived factor-1 in human endometrial stromal cells. Hum Reprod 27: 523-530, 2012.

26. Wu H, Huang S, Chen Z, Liu W, Zhou X and Zhang D: Hypoxia-induced autophagy contributes to the invasion of salivary adenoid cystic carcinoma through the HIF-1 $\alpha /$ BNIP3 signaling pathway. Mol Med Rep 12: 6467-6474, 2015.

27. Shelby SJ, Angadi PS, Zheng QD, Yao J, Jia L and Zacks DN: Hypoxia inducible factor $1 \alpha$ contributes to regulation of autophagy in retinal detachment. Exp Eye Res 137: 84-93, 2015.

28. Huang R, Xu Y, Wan W, Shou X, Qian J, You Z, Liu B, Chang C, Zhou T, Lippincott-Schwartz J and Liu W: Deacetylation of nuclear LC3 drives autophagy initiation under starvation. Mol Cell 57: 456-466, 2015.

29. Nazio F, Strappazzon F, Antonioli M, Bielli P, Cianfanelli V, Bordi M, Gretzmeier C, Dengjel J, Piacentini M, Fimia GM and Cecconi F: mTOR inhibits autophagy by controlling ULK1 ubiquitylation, self-association and function through AMBRA1 and TRAF6. Nat Cell Biol 15: 406-416, 2013.

30. Lin X, Li S, Zhao Y, Ma X, Zhang K, He X and Wang Z: Interaction domains of p62: A bridge between p62 and selective autophagy. DNA Cell Biol 32: 220-227, 2013.

31. Liu H, Zhang Z, Xiong W, Zhang L, Xiong Y, Li N, He H, Du Y and Liu Y: Hypoxia-inducible factor- $1 \alpha$ promotes endometrial stromal cells migration and invasion by upregulating autophagy in endometriosis. Reproduction 153: 809-820, 2017. 